\title{
Alterações histológicas e transporte de eletrólitos após administração de contraste de alta osmolaridade
}

\section{Histological changes and transportation of electrolytes after administration of contrast of high osmolarity}

Paula Letícia Braga e Silva1. Pedro Henrique Sá Costa ${ }^{1}$. Antônio Rafael Coelho Jorge ${ }^{1}$. João Alison de Moraes Silveira $^{1}$. Francisco Assis Nogueira Junior ${ }^{1}$. Janaina Serra Azul Monteiro Evangelista ${ }^{2}$. Helena Serra Azul Monteiro'.

1 Universidade Federal do Ceará (UFC), Fortaleza, Ceará, Brasil. 2 Universidade Estadual do Ceará (UECE), Fortaleza, Ceará, Brasil.

\section{RESUMO}

Objetivos: avaliar possíveis alterações renais causadas pelo contraste ioxitalamato de meglumina. Métodos: o contraste ioxitalamato de meglumina na dose de $5 \mathrm{~mL} / \mathrm{Kg}(\mathrm{n}=8)$ ou solução salina $0,9 \%$ na dose de $5 \mathrm{~mL} / \mathrm{Kg}(\mathrm{n}=5)$ foram administrados por via intravenosa em ratos Wistar machos (250-350g). Os animais foram mantidos em gaiolas metabólicas, e após 72 horas a função renal foi avaliada através de proteinúria, osmolaridade, ureia, creatinina, $\mathrm{Na}^{+}, \mathrm{K}^{+} \mathrm{e} \mathrm{Cl}$ plasmáticos. Os dados foram comparados por teste $\mathrm{t}$ de Student $(p<0,05)$. Resultados: observamos redução do peso corpóreo e aumento da osmolaridade plasmática. Não foi observado variação na diurese de 24 horas. Em relação aos parâmetros bioquímicos, o contraste não alterou os parâmetros renais (creatinina, ureia, proteinúria e $\mathrm{Na}^{+} \mathrm{e} \mathrm{K}^{+}$). Entretanto, o $\mathrm{Cl}^{-}$plasmático diminuiu nos animais do grupo contraste. Foram observadas mudanças histológicas significativas no grupo contraste, tais como degeneração glomerular, infiltrado inflamatório, deposição de material proteico na luz tubular, degeneração hidrópica da célula epitelial tubular, tumefação tubular e redução do interstício renal. Conclusão: esses resultados demonstram que apesar do contraste induzir alterações histológicas, não foi capaz de modificar a função renal.

Palavras-chave: Lesão renal aguda. Meios de contraste. Histologia.

\section{ABSTRACT}

Objects: The aim of this study was to evaluate possible renal disorders caused by Ioxithalamate Meglumine contrast. Methods: Ioxithalamate Meglumine at a dose of $5 \mathrm{ml} / \mathrm{kg}(\mathrm{n}=8)$ or $0.9 \%$ saline at a dose of $5 \mathrm{ml} / \mathrm{kg}(\mathrm{n}=5)$ were administered intravenously in male Wistar rats (250-350g). After a 72-hour, they were kept in metabolic cages and renal function was assessed by dosages of proteinuria, urea, creatinine, $\mathrm{Na}^{+}, \mathrm{K}^{+}$and $\mathrm{Cl}^{-}$in plasma. Data were compared using Student's $\mathrm{t}$ test, $(\mathrm{p}<0.05)$. We observed reduction of body weight and increase of plasma osmolarity. No variation in diuresis of 24 hours was observed. Results: Regarding the biochemical parameters, the contrast did not alter the renal parameters (creatinine, urea, proteinuria and $\mathrm{Na}^{+}$and $\mathrm{K}^{+}$). However, $\mathrm{Cl}^{-}$plasmatic decreased in the animals in the contrast group. Significant histological changes were observed in the contrast group such as glomerular degeneration, inflammatory infiltrate, deposition of protein material in tubular lumen, tubular epithelial cell hydropic degeneration, tubular tumefaction and reduction of the renal interstice. Conclusion: These results demonstrate that despite the contrast induces histological changes, it was not able to modify renal function.

Keywords: Acute kidney injury. Contrast media. Histology.

Autor correspondente: Paula Letícia Braga e Silva, Rua Coronel Nunes de Melo, 1000, Rodolfo Teófilo, Fortaleza, Ceará. CEP: 60416-000. Telefone: +55 85 3366-8248. E-mail: paulaleticia@alu.ufc.br

Conflito de interesses: Não há qualquer conflito de interesses por parte de qualquer um dos autores.

Recebido em: 08 Mai 2018; Revisado em: 12 Jul 2018; Aceito em: 11 Ago 2018. 


\section{INTRODUÇÃO}

Nas práticas clínica e cirúrgica atuais, o uso de contrastes iodados constitui uma alternativa importante na investigação de patologias em diversas áreas da medicina, ${ }^{1}$ estando entre as medicações mais prescritas na medicina diagnóstica. ${ }^{2} \mathrm{~A}$ principal ação desses compostos está na capacidade de alterar a densidade dos raios- $\mathrm{X}$ ao serem introduzidos no tecido alvo, permitindo a visualização de detalhes das estruturas e órgãos internos. ${ }^{3}$ Entre os exames que utilizam os produtos de contraste iodado, incluem-se angiografia, urografia intravenosa, tomografia computadorizada e mielografia. ${ }^{4}$

A nefropatia induzida por meios de contraste (NIMC) é uma importante complicação decorrente destes exames e é definida por aumento relativo maior que $25 \%$ no valor basal de creatinina ou por aumento absoluto igual ou maior que $0,5 \mathrm{mg} / \mathrm{dL}$ que ocorrem após exposição a um contraste radiológico endovenoso nas 48 horas seguintes. ${ }^{5}$ A fisiopatologia da NIMC não é totalmente conhecida, mas já foram identificados fatores tubulares e vasculares. Estima-se que seja uma combinação de toxicidade tubular direta do contraste e de isquemia medular renal. ${ }^{6,7}$

O presente estudo se propõe a avaliar a função renal in vivo, após a administração de contraste iodado de alta osmolaridade (ioxitalamato de meglumina) no intuito de investigar possíveis alterações causadas pelo mesmo, mimetizando os eventos que ocorrem na clínica em pacientes que necessitam da utilização de contraste.

\section{MATERIAIS E MÉTODOS}

Foram utilizados ratos albinos da linhagem Wistar, machos, pesando entre $250 \mathrm{~g}$ e $300 \mathrm{~g}$, pertencentes ao biotério da Unidade de Pesquisas Clínicas do Departamento de Fisiologia e Farmacologia da Universidade Federal do Ceará. Os protocolos experimentais foram aprovados pelo Comitê de Ética no Uso de Animais (CEUA) da Universidade Federal do Ceará sob o número 108/11.

Todos os animais foram submetidos a um período de adaptação de 24 horas em gaiolas metabólicas individuais. Após esse período, os animais foram separados em dois grupos e administrou-se, em dose única por via intravenosa, salina (Controle; $\mathrm{n}=5$ ) ou ioxitalamato de meglumina (Contraste; $5 \mathrm{~mL} / \mathrm{Kg})(\mathrm{n}=8)$. Após 72 horas, os animais foram sacrificados para coleta de sangue e extração dos rins.

Para cada animal, os seguintes procedimentos foram adotados ao início (t0) e ao final (t72) do experimento: avaliação do peso corporal e volume urinário; avaliação do peso do rim do animal pós-eutanásia; coleta de sangue e urina para dosagens bioquímicas (ureia, creatinina e eletrólitos séricos e creatinina e proteínas urinárias) e análise histológica do órgão.

A coleta das amostras de sangue foi realizada por punção na veia cava, em eppendorffs heparinizados, que foram centrifugados a 3500 rotações por minuto (rpm). A seguir, o plasma foi separado e estocado em freezer $-20^{\circ} \mathrm{C}$ até a realização das análises.
Para a determinação quantitativa de creatinina no soro e na urina foi utilizado o método de Jaffé modificado, através do kit Creatinina K (Ref: 96) da Labtest ${ }^{\circledR}$. Os resultados foram expressos em $\mathrm{mL} / \mathrm{min}$.

Para determinar a ureia quantitativamente no soro dos animais, utilizou-se o Kit ureia CE (Ref: 27) Labtest $^{\mathbb{}}$, seguindo o princípio do método colorimétrico do azul de indofenol. Ao fim das dosagens, os resultados foram expressos em mg/dL.

Para a dosagem de proteína na urina, foi utilizado o Kit Sensitprot (Ref: 36) da Labtest ${ }^{\circledR}$, através do método colorimétrico do vermelho de piragalol. Os resultados foram expressos em miligrama por 24 horas ( $\mathrm{mg} / 24 \mathrm{~h})$, utilizando-se do valor do volume urinário de cada animal nas gaiolas metabólicas.

Utilizando o método eletrodo íon seletivo, por meio do analisador de eletrólitos 9180 (Roche, Brasil), foram mensurados os níveis plasmáticos de $\mathrm{Na}^{+}, \mathrm{K}^{+} \mathrm{e} \mathrm{Cl}^{-}$.

Imediatamente após eutanizar os animais, foi realizada a extração e pesagem dos rins. Em seguida, amostras de tecido dos rins foram armazenadas em 10\% de formalina tamponada durante 24 horas, em seguida, etanol (70\%). Os fragmentos foram submetidos a desidratação, diafanização e corte a uma espessura de $5 \mu \mathrm{m}$. As lâminas foram coradas com hematoxilina-eosina (HE) e analisadas utilizando uma óptica trinocular. Os cortes histológicos foram obtidos utilizando micrótomo de impacto (Poicut S, Leica, Alemanha), equipado com navalha detunsgstênio de $16 \mathrm{~cm}$, tipo D (Leica, Alemanha).

Para análise estatística todos os dados foram expressos como média \pm erro padrão da média e analisados através do programa GraphPadPrism 5.0, utilizando análise teste t de Student.

\section{RESULTADOS}

Observou-se uma diminuição significativa no peso corpóreo dos dois grupos em estudo após decorrido o tempo de 72 horas em gaiola metabólica $(\mathrm{p}=0,0009)$ (Figura 1a). Entretanto, este mesmo efeito não pôde ser observado em relação ao peso do rim isoladamente (Controle 0,836 $\pm 0,007 \mathrm{~g}$ vs Contraste $0,808 \pm 0,021 \mathrm{~g}$ ), demonstrando que o contraste ioxitalamato de meglumina não foi capaz de alterar o peso renal dos animais.

Conforme observado na Figura 1b, a osmolaridade plasmática apresentou um aumento significativo no grupo contraste em relação ao controle (Controle 318,7 $\pm 8,09 \mathrm{mmol} / \mathrm{Kg}$ vs Contraste $352 \pm 8,38 \mathrm{mmol} / \mathrm{Kg}) ;(\mathrm{p}=0,0533)$, enquanto que na Figura 1c não houve diferença significativa no volume urinário de 24 horas dos animais que receberam o contraste ioxitalamato de meglumina em relação ao grupo controle (Controle 10,43 $\pm 3.675 \mathrm{~mL} / 24 \mathrm{hrs}$ vs Contraste 4,338 \pm 1.57 $\mathrm{mL} / 24 \mathrm{hrs})(\mathrm{p}=0,1192)$. 
Figura 1. Avalição dos parâmetros gerais, sendo em a) peso corpóreo dos animais após 72 horas em gaiola metabólica, em b) a osmolaridade plasmática dos animais que receberam contraste Ioxitalamato de Meglumina e os que receberam salina e em c) o volume urinário de 24 horas de animais que receberam o contraste Ioxitalamato de Meglumina e os que receberam salina.

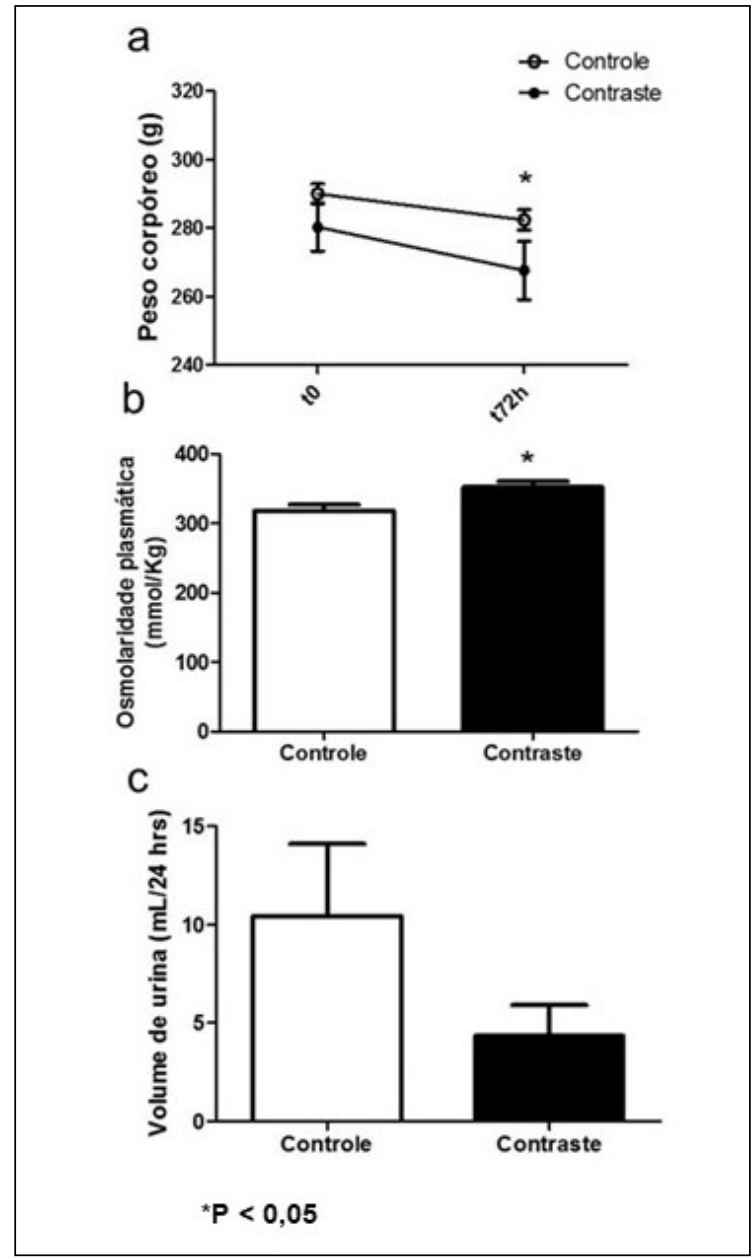

Nota: Os valores da figura expressam a média \pm erro padrão da média (E.P.M) do peso dos animais em cada grupo, em dois tempos diferentes. Grupos: Controle: que recebeu somente salina $(5 \mathrm{ml} / \mathrm{kg})$; Contraste: que recebeu o contraste Ioxitalamato de Meglumina $(5 \mathrm{~mL} / \mathrm{kg})$. T0: representa o tempo precedente aos animais serem colocados na gaiola metabólica; T72: último dia do protocolo experimental, 30 minutos antes do sacrifício dos animais. A análise estatística foi realizada por teste $\mathrm{t}$ de Student pareado para a análise do peso corpóreo e não pareado para as demais análises. Houve significância entre os grupos $(p<0,05)$.

A Figura 2 evidencia que o modelo experimental do presente estudo não foi eficaz em induzir aumento significativo dos níveis de creatinina (2a), ureia (2b) e proteínas urinárias (2c) dos animais em que foi administrado o contraste.

No presente trabalho, foram determinados os níveis séricos de $\mathrm{Na}^{+}, \mathrm{K}^{+}$e $\mathrm{Cl}^{-}$. Os achados mostraram que o contraste ioxitalamato de megluminal levou a uma diminuição significativa nos níveis séricos de $\mathrm{Cl}^{-}$dos animais $(\mathrm{p}=0,0339)$ (Figura 3c), sem causar diferenças significativas nos níveis séricos de $\mathrm{Na}^{+}$(Figura 3a) e potássio (Figura 3b) entre os grupos em estudo.

Alterações histopatológicas foram observadas nos três segmentos do rim: glomérulos, túbulos e interstício (Figura 4). A análise histológica evidenciou alterações significativas a nível glomerular, com presença de glomérulos degenerados; além de infiltrado inflamatório celular linfoplasmocitário; os segmentos tubulares apresentaram degeneração hidrópica e tumefação tubular; deposição proteica na luz tubular; redução do interstício tubular e degeneração hidrópica da célula epitelial tubular, no grupo tratado com o meio de contraste (4a). No grupo controle, não houve qualquer indício de citotoxicidade (4b).

Figura 2. Avaliações bioquímicas, sendo em a) os níveis plasmáticos de creatinina em animais que receberam contraste Ioxitalamato de Meglumina e os que receberam salina, em b) níveis de ureia sérica em ratos nos dois grupos em estudo e em c) níveis de proteína na urina de 24 horas.

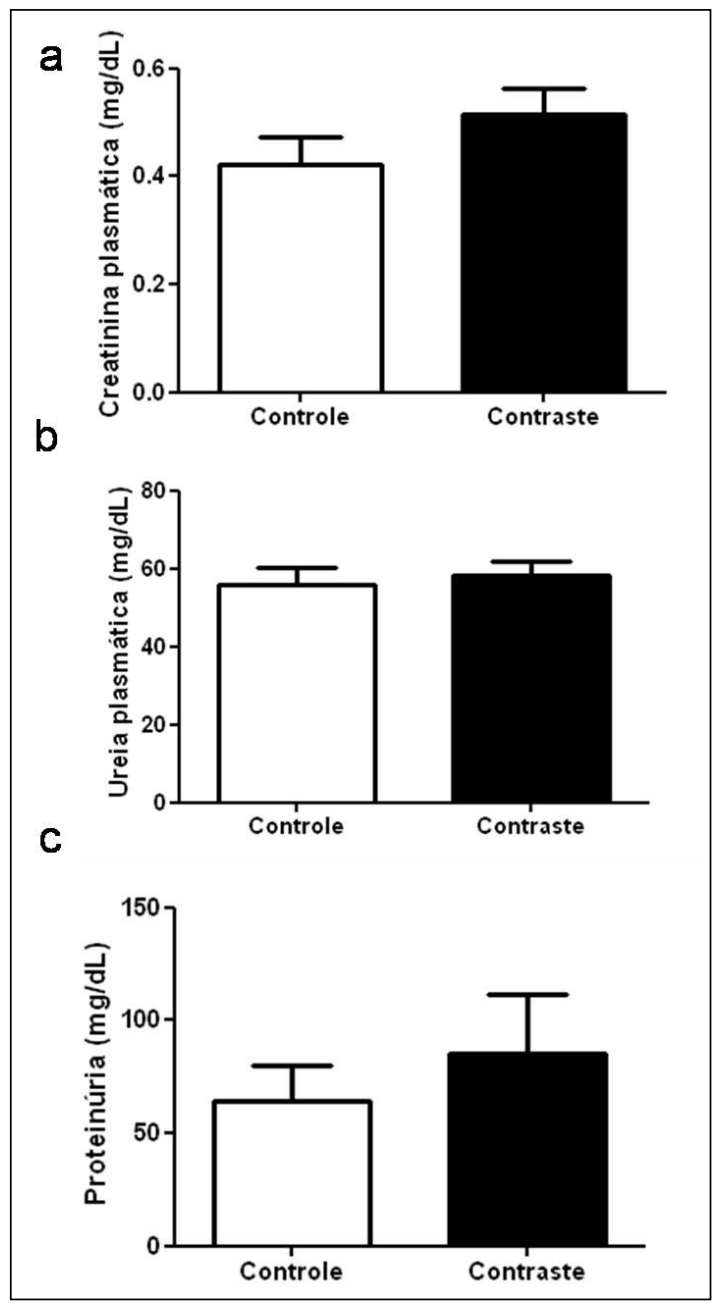

Nota: Os valores da figura expressam a média \pm E.P.M dos níveis plasmáticos de creatinina em cada grupo. Grupos: Controle: que recebeu somente salina $(5 \mathrm{ml} /$ $\mathrm{kg}$ ); Contraste: que recebeu o contraste Ioxitalamato de Meglumina $(5 \mathrm{~mL} / \mathrm{kg})$. A análise estatística foi realizada por teste $t$ de Student. Não foram observadas diferenças significativas entre os grupos. 
Figura 3. Níveis plasmáticos de sódio em a, potássio em b e cloreto em c dos animais.

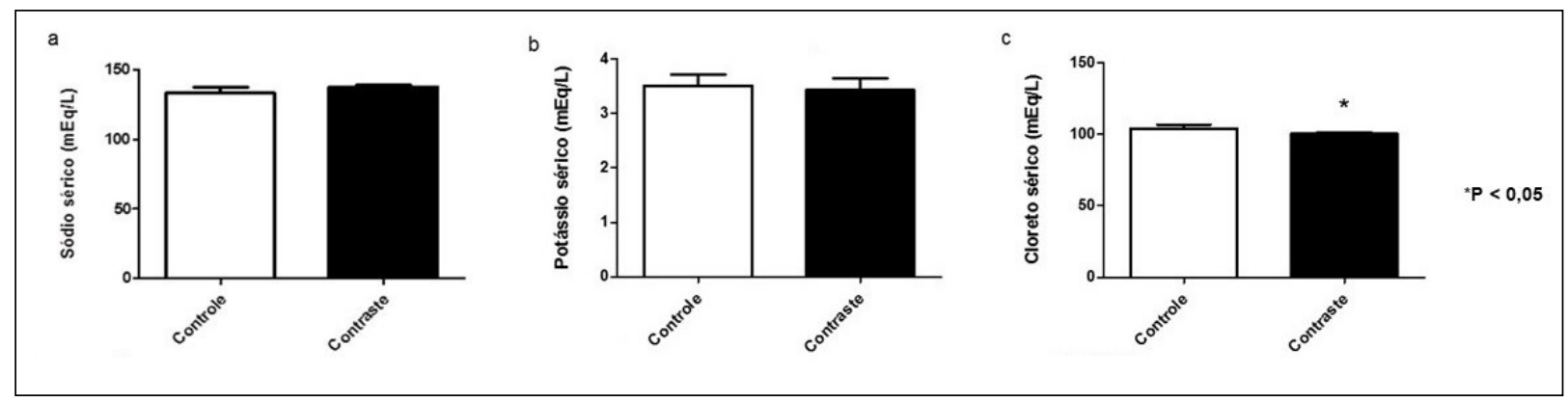

Nota: Os valores da figura expressam a média \pm E.P.M dos níveis plasmáticos de $\mathrm{Na}^{+}, \mathrm{K}^{+}$e $\mathrm{Cl}^{-}$em cada grupo. Grupos: Controle: que recebeu somente salina $(5 \mathrm{~mL} / \mathrm{kg})$; Contraste: que recebeu o contraste Ioxitalamato de Meglumina $(5 \mathrm{~mL} / \mathrm{kg})$. A análise estatística foi realizada por teste $t$ de Student. Foi observada diferença significativa no grupo contraste em relação ao grupo controle para os níveis plasmáticos do íon cloreto.

Figura 4. Fotomicrografias de rim de rato do grupo controle $(5 \mathrm{~mL} / \mathrm{kg})$ e grupo contraste Ioxitalamato de Meglumina ( $5 \mathrm{~mL} / \mathrm{kg})$.

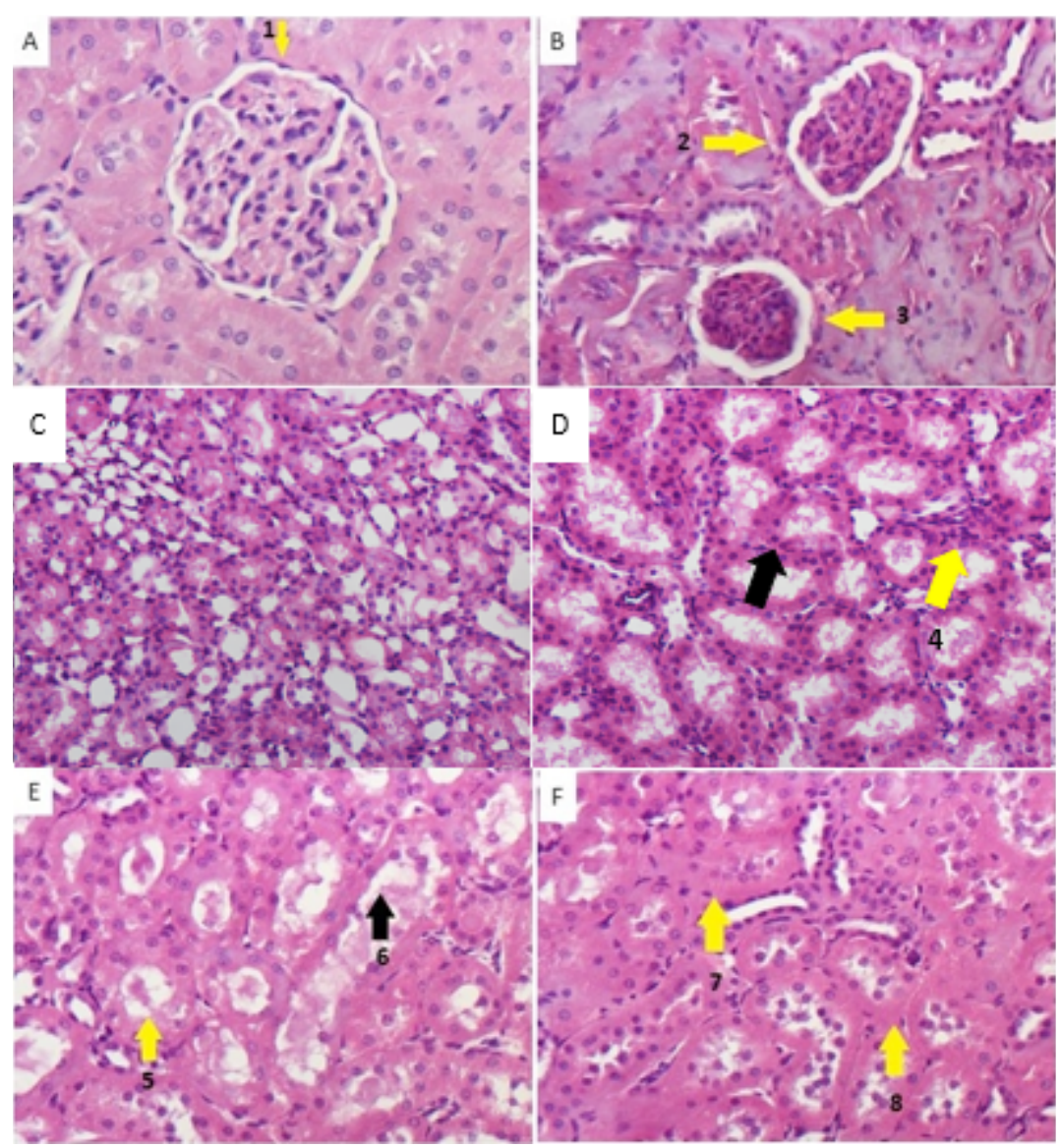

Nota: As fotomicrografias A e B representam, respectivamente, glomérulos renais dos grupos controle e contraste. A seta amarela na imagem A representa um glomérulo normal e as setas amarelas na imagem B representam glomérulos degenerados. Imagens observadas em microscópio óptico em aumento de 400x. A imagem C representa medula renal normal no grupo controle. Na imagem $\mathrm{D}$, a seta em preto aponta para a tumefação intersticial e a seta amarela indica infiltração celular linfoplasmocitária. Imagens observadas em microscópio óptico em aumento de 400x. As imagens E e F representam túbulos renais do grupo contraste. A seta em amarelo na imagem E representa depósito de material proteico na luz tubular e a seta preta demonstra degeneração hidrópica e tumefação celular. Na imagem F, as setas em amarelo representam tumefação tubular e redução do interstício renal. Imagens observadas em aumento de 400x. Microscópio Nikon Eclipse Nis, Software Nis 4.0. 


\section{DISCUSSÃO}

A administração do meio de contraste de alta osmolaridade demonstrou uma tendência na redução do fluxo urinário, que pode ser explicado devido ao aumento da viscosidade do sangue total. ${ }^{8}$ Além disso, observamos uma redução significativa do peso corporal dos animais, provavelmente devido ao aumento das condições estressantes a que foram submetidos. ${ }^{9}$

Nesse estudo, não foram observadas mudanças no funcionamento renal pela realização das dosagens bioquímicas, presumindo-se que o contraste nesta dose e tempo de tratamento não foi capaz de causar nefropatia. Alterações significativas na concentração de cloreto sérico e nenhuma alteração nas concentrações séricas de sódio e potássio podem ser reflexo de mecanismo de autorregulação renal que operam rapidamente para restabelecer o aumento da viscosidade sanguínea causada pelo contraste. $\mathrm{O}$ aumento da osmolaridade estimula a liberação do hormônio antidiurético (ADH) que atua rapidamente no rim restabelecendo o equilíbrio hidroeletrolítico. ${ }^{10}$ Sabe-se que todo sistema orgânico possui uma reserva funcional que habitualmente não é utilizada, porém, de acordo com novas situações fisiológicas essa reserva pode modificar as habilidades renais funcionais. Os mecanismos compensatórios intra renais são elementos fundamentais na determinação de reserva renal, que são evidenciadas tanto em nefropatia aguda como crônica. ${ }^{11}$

Alterações estruturais nos túbulos renais, distúrbios funcionais tubulares e modificações na hemodinâmica renal são alguns mecanismos descritos como relevantes na patogênese causada pelos meios de contraste. ${ }^{12}$ Os parâmetros histopatológicos,

\section{REFERÊNCIAS}

1 Persson PB. Contrast medium-induced nephropathy - editorial. Nephrol Dial Transplant. 2005;20(Sup 1):i1.

2 McCullough PA, Choi JP, Feghali GA, Schussler JM, Stoler RM, Vallabahn RC, et al. Contrast-induced acute kidney injury. J Am Coll Cardiol. 2017;68(13):1878.

3 Sugawara AM, Daros KA. Manual de meio de contraste em Rx. São Paulo: Centro Universitário São Camilo; 2004.

4 Santos AP, Galvão AM, Tavares A, Ferreira S. Produtos de contrastes iodados. Acta Med Port. 2009;22(3):261-74.

5 Rihal CS, Textor SC, Grill DE, Berger PB, Ting HH, Best PJ, et al. Incidence and prognostic importance of acute renal failure after percutaneous coronary intervention. Circulation. 2002;105(19):2259-64.

6 Costa JA, Vieira OM Neto, Moysés M Neto. Insuficiência renal aguda. Medicina (Ribeirão Preto). 2003;36:307-24.

7 Ultramari FT, Bueno Rda R, da Cunha CL, de Andrade PM, Nercolini DC, Tarastchuk JC, et al. Contrast media-induced nephropathy following diagnostic and therapeutic cardiac catheterization. Arq Bras Cardiol. 2006;87(3):378-90. como degeneração glomerular, tumefação intersticial e infiltração celular linfoplasmocitária, correlacionaram-se positiva e significativamente com os achados na literatura.

Os efeitos citotóxicos observados podem ter sido resultado do aumento da osmolaridade sanguínea pelo contraste, que ativa respostas de feedback túbuloglomerular e que acarreta vasoconstrição. ${ }^{13}$ Essa vasoconstrição da arteríola aferente diminui a oferta de $\mathrm{O}_{2}$ aos túbulos renais e gera hipóxia, resultando em morte celular. Além disso, o aumento da viscosidade sanguínea pode contribuir ainda mais para essa hipóxia, por reduzir a oferta de $\mathrm{O}_{2}$ ao tecido renal. ${ }^{14}$

Citotoxicidade direta ou geração de radicais livres de oxigênio é um dos efeitos do contraste de alta osmolaridade, causando injúria renal. ${ }^{15}$ As alterações histológicas causadas pelo contraste demonstram que esse composto possui capacidade de causar lesão nas células renais, entretanto, os danos não foram suficientes para induzir a NMC devido ao fato dos animais não possuírem nenhum dos fatores de risco para desenvolvimento do NIMC, que incluem: desidratação, diabetes mellitus, IRC preexistente, insuficiência cardíaca congestiva ${ }^{16}$ e também pela dose utilizada ser a mesma utilizada na clínica, não sendo, portanto, capaz de causar nefropatia em ratos sadios e com funções renais preservadas.

\section{CONCLUSÃO}

Os resultados das dosagens bioquímicas realizadas no presente estudo sugerem que não houve alterações significativas que comprometessem a função renal. Entretanto, houve alterações na histologia renal e transporte de cloreto, o que nos demonstra que o contraste pode ser lesivo ao rim, principalmente quando associado a fatores de risco pré-existentes que o potencializem.

8 Wong GT, Irwin MG. Contrast-induced nephropathy. Br J Anaesth. 2007;99(4):474-83.

9 Kalliokoski O, Jacobsen KR, Darusman HS, Henriksen T, Weimann A, Poulsen HE, et al. Mice do not habituate to metabolism cage housing-A three week study of male BALB/c mice. PlosOne. 2013;8(3):58460.

10 Knepper MA. Molecular physiology of urinary concentrating mechanism: regulation of aquaporin water channels by vasopressina. Am J Physiol. 1997;272(41):F3-12.

11 Douglas CR. Patofisiologia de sistema: renal. São Paulo: Robe; 2001. Capítulo 1, Homeostase renal; p. 62-7.

12 Ribeiro L. Avaliação da produção de óxido nítrico em cultura de célular da artéria renal de ratos expostos aos diferentes meios de contraste radiológico [dissertação]. São Paulo: Universidade Federal de São Paulo; 2003. 97 f.

13 Santos AP, Gaivão AM, Tavares A, Ferreira S. Produtos de contraste iodados. Acta Med Port. 2009;22:261-74.

14 Andrade L, Seguro AC. Revisão/atualização em insuficiência 
renal aguda: nefrotoxicidade induzida pelo radiocontraste. J Bras Nefrol. 1997;19(2):208-11.

15 Rudnick MR, Tumlin JA. Pathogenesis, clinical features, and diagnosis of contrast-induced nephropathy [Internet]. 2011 [Acesso em: 03 abr 2018]. Disponível em: https://www.uptodate.com/ contents/pathogenesis-clinical-features-and-diagnosis-of-contrastinduced-nephropathy

16 Waybill MM, Waybill PN. Contrast media-induced nephrotoxicity: identification of patientes at risk and algorithms for prevention. J Vasc Interv Radiol. 2001;12:3-9.

\section{Como citar:}

Silva PL, Costa PH, Jorge AR, Silveira JA, Nogueira FA Junior, Evangelista JS, et al. Alterações histológicas e transporte de eletrólitos após administração de contraste de alta osmolaridade. Rev Med UFC. 2019 abr-jun;59(2):21-26. 